Scripta METALLURGICA et MATERIALIA
Vol. 26, pp. $1529-1534,1992$

Printed in the U.S.A.
Pergamon Press Ltd. All rights reserved

VIEWPOINT SET No. 18

\title{
SURFACE FILM EFFECTS DURING CYCLIC DEFORMATION OF DISLOCATION-MOBILITY-LIMITED MATERIALS
}

\author{
K.J. Bowman*, S.E. Hartfield-Wünsch** and R. Gibala ***
}

\author{
* School of Materials Engineering, Purdue University, West Lafayette, IN 47907 \\ ** General Motors Technical Center, 30300 Mound Road, Warren, MI 48090-9040 \\ *** Department of Materials Science and Engineering, University of Michigan, Ann Arbor, MI 48109-2136
}

\author{
(Received September 13, 1991)
}

(Revised October 21, 1991)

Introduction

Let us define a dislocation-mobility-limited material simply as one for which the intrinsic glissile mobility of dislocations is strongly temperature dependent because it is limited by stress-assisted thermally activated process (1). The glide resistance may result from the Peierls stress of specific dislocation components, as for $1 / 2<111>$ screw dislocations in bcc metals deformed at $T \leq 0.15 T_{m}$, where $T_{m}$ is the absolute melting temperature (2), from intrinsic locking processes, such as Kear-Wilsdorf-like locks on screw dislocations in $\mathrm{Ni} 3 \mathrm{Al}$ deformed at temperatures below the yieldstress maximum (3), or from any intrinsic dislocation process which controls the temperature and strain rate dependence of macroscopic flow. In this paper, we demonstrate that surface films can enhance the cyclic plasticity of such dislocation-mobility-limited materials in a similar manner to monotonic deformation of these materials. The results on cyclic deformation provide an unusual opportunity to explore surface film effects under essentially constant dislocation structure, which is not as easily accomplished in monotonic experiments. In cyclic deformation, the dislocation motions characteristic of microstrain and macrostrain processes can be sustained to large accumulated strains without failure or even initiation of cracks. The film effects can be explored for different types of dislocation motion under the nearly steady-state conditions of cyclic saturation.

Our group began investigation of surface film effects on crystal plasticity of dislocation-mobility-limited materials during monotonic deformation more than fifteen years ago. The initial observations showed that surface oxide films on single crystals of the bec metals $\mathrm{Nb}$ and $\mathrm{Ta}$ can significantly reduce the flow stress and increase the plasticity at low homologous temperatures (4). At these temperatures, plasticity of the bec metals is controlled by the large Peierls-like barrier associated with the non-planar core structure of $1 / 2<111>$ screw dislocations. Non-screw dislocations have a planar core structure, and their mobility is much higher at the same temperature. Film softening occurs because the stressed film-substrate interface, under conditions of elastic and plastic constraint, acts as a source for the high-mobility non-screw dislocations. Hence plastic flow occurs at reduced stresses compared to the uncoated materials, for which macroscopic deformation is controlled by the less mobile screw dislocations. Operation of surface-film softening processes is demonstrated effectively by "film on-film off" experiments (4) and by experiments in which surfaces of welldefined orientations relative to the operative Burgers vector are selectively coated (5). These observations of surface film softening in monotonic deformation of single crystals of $\mathrm{Nb}$ and $\mathrm{Ta}$ were subsequently extended to Mo and $\mathrm{W}(6,7)$ and to Fe (8). Polycrystalline forms of the bcc metals and other dislocation-mobility-limited materials also exhibit surface film softening $(7,9,10)$.

More recently, observations of surface film softening in monotonic deformation have been extended to single crystals and polycrystalline materials of the $\mathrm{B}_{2}$ ordered alloys $\mathrm{FeAl}$ and $\mathrm{NiAl}$ (9-12). At low homologous temperatures, these ordered alloys deform primarily by the motion of $\langle 111\rangle$ and $<001\rangle$ dislocations, respectively. FeAl has a relatively low APB energy, and deformation by $<111>$ dislocations appears to exhibit a comparable difference in edge and screw dislocation mobility to that predicted and observed for bcc metals $(10,13)$. The $<111>$ screw dislocation is dissociated into a non-planar configuration, and the $\langle 111\rangle$ edge dislocation is characterized by a planar core structure. For this material, surface film softening probably occurs in the manner of bcc metals: excess densities of the higher mobility edge dislocitions are generated at the film-substrate interface, effecting a reduced flow stress and increased ductility. By contrast, $\mathrm{NiAl}$ and some $\mathrm{Ni}-\mathrm{Fe}-\mathrm{Al}$ ordered alloys we report on here have a relatively higher $\mathrm{APB}$ energy and deform by motion of $\langle 0(0) 1>$ dislocations, except in the limit of single crystals of the "hard" $<001>$ orientation, which deform by $<111>$ dislocations because of Schmid factor considerations (14). There appears to be no particularly large difference in mobility of $\langle 0(0) 1\rangle$ edge and screw dislocations in $\mathrm{NiAl}(13,15,16)$. Theoretical work on $<001\rangle$ dislocation core structures suggests that all $<001>$ dislocations have non-planar cores, such that no one dislocation type limits plastic flow at low temperatures $(16)$. TEM observations $(10,17,18)$ suggest that, if anything, mixed dislocations may have the 
lowest mobility of the various dislocation components in $\mathrm{NiAl}$ and $\mathrm{Ni}-\mathrm{Fe}-\mathrm{Al}$ alloys. Consequently, surface film softening in $\langle(0) 1>$ dislocation-containing materials is thought to occur from a general dislocation pumping process occurring at the film-substrate interface, which results in an effective increase in the overall mobile dislocation density available to effect plastic flow. Such results are analogous to reduced flow stresses observed by Ruddle and Wilsdorf for nickel-plated single crystals of copper many years ago (19).

The specific purpose of this paper is to demonstrate some of the ways in which surface-film-enhanced plasticity that is now well documented in monotonic deformation studies of dislocation-mobility-limited materials can be manifested during cyclic deformation of these same materials. As in previous studies of monotonic deformation, we focus on relatively low homologous temperatures, typically near or below the ductile-to-brittle fracture transition temperature. We use results for single crystal W and polycrystalline $\mathrm{Fe}-\mathrm{Al}$ and $\mathrm{Ni}-\mathrm{Fe}$-Al ordered alloys as examples of the effects. The use of the two different ordered alloys allows comparison of $\mathbf{B}_{2}$ ordered alloys which deform by $\langle 111\rangle$ and $\langle 001\rangle$ dislocations, respectively. The former material meets the von Mises criterion for grain boundary compatibility requiring five independent slip systems for homogeneous deformation of a polycrystalline ensemble, whereas the latter does not. For all three of these materials, room temperature is a conveniently low homologous temperature for which ductility enhancement from surface film effects in cyclic deformation can be examined.

\section{Experimental}

Details of the experimental procedures are given elsewhere $(10,20)$ and are described only briefly here. High purity W single crystals with a [213] rod axis were prepared by a combination of electron-beam zone melting of $99.95 \%$ pure polycrystalline stock in a vacuum of $\sim 10^{-4} \mathrm{~Pa}$ followed by ultra-high vacuum outgassing at $2800 \mathrm{~K}$ for $2-3$ days. From resistivity and microhardness data, the crystals are estimated to have less than $10 \mathrm{ppma}$ residual solutes. The fatigue specimens were typically $70 \mathrm{~mm}$ long and $2 \mathrm{~mm}$ in gage diameter, with a gage length of about $10 \mathrm{~mm}$. The polycrystalline $\mathrm{Ni}-\mathrm{Fe}-\mathrm{Al}$ and $\mathrm{Fe}$-Al alloys of nominal compositions $\mathrm{Ni}-20 \mathrm{Fe}-30 \mathrm{Al}$ and $\mathrm{Fe}-40 \mathrm{Al}$ (in at.\%) were prepared as extrusions made from rapidly-solidified powders. Extrusion temperatures were $1200 \mathrm{~K}-1400 \mathrm{~K}$ and $1140 \mathrm{~K}-1250 \mathrm{~K}$, respectively, at an extrusion ratio of 16:1. Grain sizes were $15-30 \mu \mathrm{m}$ and $20-35 \mu \mathrm{m}$, respectively, with the smaller grain sizes corresponding to the lower extrusion temperatures for both materials. Most specimens were $67 \mathrm{~mm}$ long and $3 \mathrm{~mm}$ in gage diameter, with a gage length of $12 \mathrm{~mm}$. The surface films selected for use in the present experiments on cyclic deformation are those which have been shown to effect surface film softening in monotonic deformation. For $\mathrm{W}$, specimens were anodized at room temperature in $0.1 \mathrm{M} \mathrm{H}_{2} \mathrm{SO}_{4}$ at room temperature at a potential of $40 \mathrm{~V}$, which produces an $\sim 80 \mathrm{~nm}$ anodic oxide film. The film could be removed from the specimen for in situ or post situ experiments by dissolution in $0.05 \mathrm{M} \mathrm{NaOH}$. Nickel coatings $30-250 \mathrm{~nm}$ in thickness, as measured by Rutherford backscattering (RBS), were applied at room temperature to the $\mathrm{Ni}-\mathrm{Fe}-\mathrm{Al}$ and $\mathrm{Fe}-\mathrm{Al}$ alloys by a standard electroless nickel plating technique. Both the oxide and the nickel films were adherent to their respective substrate materials during both monotonic and cyclic deformation experiments and did not show significant cracking. Analyses of the growth stresses for both the oxide and nickel films suggest the substrate materials were in residual tension in the interface region of the film-substrate composites. Cyclic deformation experiments were performed in plastic strain control on a computer-controlled MTS servohydraulic machine equipped with hydraulic collet grips. All tests were performed at room temperature under ambient pressure at total strain rates, $\dot{\varepsilon}$, of about $10^{-4} / \mathrm{s}$. Plastic strain amplitudes, $\varepsilon_{\mathrm{pl}}$, ranged from $10^{-5}$ to $10^{-2}$. All of the cyclic deformation tests were complemented by comprehensive observations of the dislocation substructures $(10,20)$, but the TEM data are not reported in the present paper.

\section{Results and Discussion}

\section{Body-Centered Cubic Metals}

The cyclic deformation behavior of uncoated bcc metals at low homologous temperatures has become better understood in the last several years (21). As with monotonic deformation, there are distinct microstrain and macrostrain deformation regimes, which are readily discernible in plastic strain control experiments performed over a wide range of plastic strain amplitudes. Figure 1 gives typical results for $\mathrm{W}$ single crystals deformed at room temperature at a plastic strain rate of $3 \times 10^{-4} / \mathrm{s}$. The results are given in the form of cyclic hardening curves (peak stress versus accumulated strain) at microstrain and macrostrain levels of plastic strain amplitudes of $5 \times 10^{-5}$ and $3.75 \times 10^{-3}$, respectively. The designations $\mathbf{T}$ and $\mathbf{C}$ refer to the tensile and compression peak stresses, respectively. The cyclic stress asymmetry for $\mathrm{W}$ and other bcc metals is discussed in a separate paper (22).

The results of Fig. 1 are replotted in Fig. 2 with results for uncoated $W$ at other strain amplitudes as a curve of Young's modulus-compensated tensile saturation stress as a function of plastic strain amplitude. Also included in Fig. 2 are results for single crystals of other bcc metals $\mathrm{Nb}$, Mo and $\mathrm{Fe}$ of similar orientations at several different homologous temperatures (23-28) and a typical curve for face-centered cubic metals (e.g. Cu) over a wide range of temperatures above $0.15 T_{m}$ (29). As discussed previously (21), the cyclic deformation of uncoated bcc metals, when represented by such curves at these low homologous temperatures, is characterized by two distinct regimes of behavior corresponding to cyclic microstrain at low applied stresses and cyclic macrostrain at relatively high stresses. The strongly temperature- 
dependent plateau level of the modulus-compensated saturation stress in cyclic macrostrain of the bcc metals presented in Fig. 2 begins at lower plastic strain amplitudes as the test temperature is decreased or the cyclic plastic strain rate is increased, in similar fashion to monotonic deformation behavior of bcc metals (30). In the lower plateau regime at the lower strain amplitudes, cyclic microstrain deformation is controlled by the back and forth motion of an essentially constant density of mobile edge dislocations (28). The range of strain amplitudes over which cyclic microstrain is observed increases with increasing temperature and decreasing strain rate, as the mobility difference between edge and screw dislocations diminishes. At the higher strain amplitudes, the stress levels, which for W are as much as $600 \mathrm{MPa}$ larger than for cyclic microstrain, are sufficient to also move and obtain plasticity from the less-mobile screw dislocations, leading to the formation of a dislocation cell structure with large accumulations of loop debris. For fcc metals, with no significant difference in mobility between edge and screw dislocations, there is no comparably large change in saturation stress over the practical range of plastic strain amplitudes of $10^{-5}-10^{-2}$. The magnitude of the single plateau stress of fcc metals increases "slightly" with decreasing temperature $(31,32)$, similar to the modest temperature and strain rate dependences of the plateau stresses for cyclic microstrain of the bcc metals given in Fig. 2.

The results in Fig. 2 also include ones at room temperature for oxide-coated single crystals of W which show pronounced monotonic film softening (6). There are two major effects of surface films on the cyclic deformation of $W$ : (i) a large film-induced cyclic hardening at cyclic microstrains of the order $10^{-5}-10^{-4}$; (ii) a smaller, but well-discerned film-induced cyclic softening at cyclic macrostrains $\geq 10^{-3}$. Each effect is described separately.

Cyclic Microstrain. For plastic strain amplitudes in the microstrain regime in Fig. 2, the saturation stresses are very sensitive to the presence of surface films and the excess edge dislocation density generated by cyclic deformation processes at the film-substrate interface. The maximum difference in saturation stress for the uncoated and coated $W$ single crystals is greater than $400 \mathrm{MPa}$. The clearest demonstration of film hardening in cyclic microstrain is shown in experiments wherein the film is repeatedly applied to the surface and later removed by in situ dissolution, Fig. 3. Application of a surface film to a soft, cyclically microstrained specimen results in rapid cyclic hardening when cyclic deformation is resumed under the same conditions. The specimen hardens over $300 \mathrm{MPa}$ in 125 cycles without reaching saturation. Upon removal of the film, cyclic softening is immediate. The rate of softening is nearly equal to the prior hardening rate, and the peak stresses quickly approach the original saturation level. The rate of softening or hardening diminishes as further reapplications/removals are carried out. Nonetheless, even as the accumulated strain approaches unity, the peak stress still drops by over $100 \mathrm{MPa}$ upon film removal. The difference in visible slip features between uncoated and coated $W$ is dramatic (20). For uncoated $W<111>$ slip is prevalent on both $\{110\}$ and $\{112\}$ planes, whereas the presence of the surface film almost totally suppresses the occurrence of visible slip markings. This observation, coupled with rapid cyclic hardening upon reapplication of the film, suggests that the hardening effect is associated with the combined effects of the interaction of excess edge dislocations afforded by the surface-film-induced multiplication processes and the difficult egress of dislocations at the surface in the coated condition. Thus, a low stress level is milntained in the uncoated material by the fairly easy motion, multiplication and egress of available high mobility edge dislocations. Cyclic microstrain of the coated material produces an additional steady-state supply of mobile edge dislocations, but their enhanced interactions and restricted egress results effectively in a reduced mobile dislocation density to carry the cyclic plastic flow. The net result is the observed film-induced cyclic hardening process.

Cyclic Macrostrain. For plastic strain amplitudes in the macrostrain regime, there is a $30-50 \mathrm{MPa}$ reduction in the saturation stress for $\mid 213\}$-oriented W single crystals. The overall cyclic softening is a net result of the competing effects of cyclic hardening associated with edge and screw dislocation motion and interactions during the fatigue process and surface film softening associated with edge dislocation pumping from the film-substrate interface. The observed softening is well displayed in film on-film off experiments. In Fig. 4, we illustrate that application of a surface film during cyclic deformation of a previously uncoated single crystal produces a rapid reduction in tension and compression peak stresses at saturation toward the stress level normally observed for an initially coated material. As the accumulated strain increases to well beyond saturation, the effect of the surface film diminishes, and experiments involving film removal or application show no effects like those in Fig. 4. Note that the cyclic film softening is much larger in the tension half cycles than in the compres:ion half cycles. Observations on W crystals of other orientations confirm that the softening effect occurs primarily in tension. This result corresponds well with the greater monotonic softening effect observed in tension than in compression (33). The result is also consistent with the determination of tensile growth stresses in the W substrate due to the anodically deposited surface oxide film (34). In cyclic macrostrain, very different surface slip behavior is observed at the same accumulated strain for uncoated and coated materials (20). Surface films produce coarse, wavy slip combined with fairly straight slip bands corresponding to anomalous, low-Schmid-factor 1110\} planes, whereas fairly fine slip is observed on $\{112\}$ planes for the uncoated material. This substantial difference in surface slip features in the uncoated and coated materials suggests that surface films have a very strong effect on deformation behavior near the surface, even when the change in mechanical response is relatively small in cyclic makrostrain. 


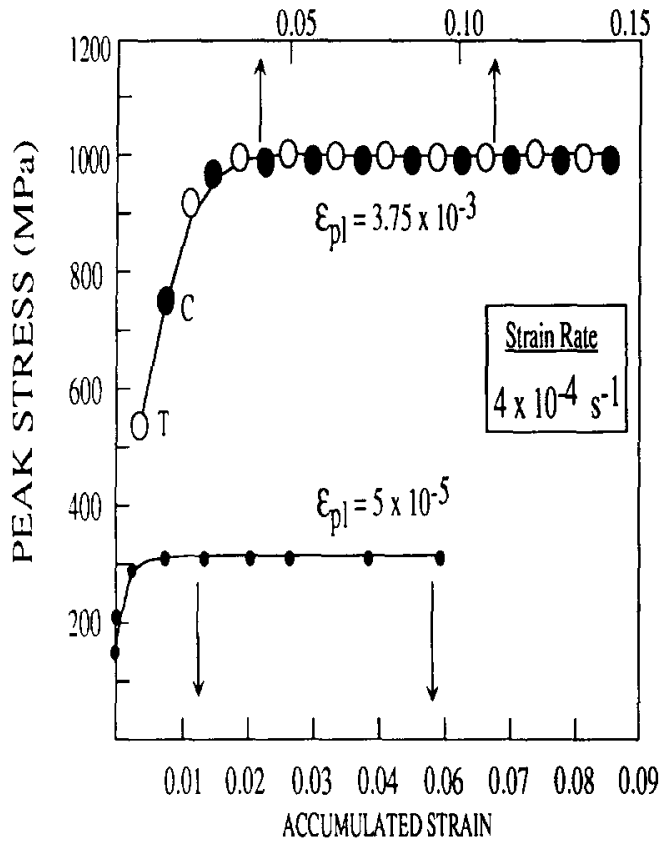

Fig. 1. Cyclic hardening of $W$ at plastic microstrains and macrostrains for [213]-oriented single crystals.

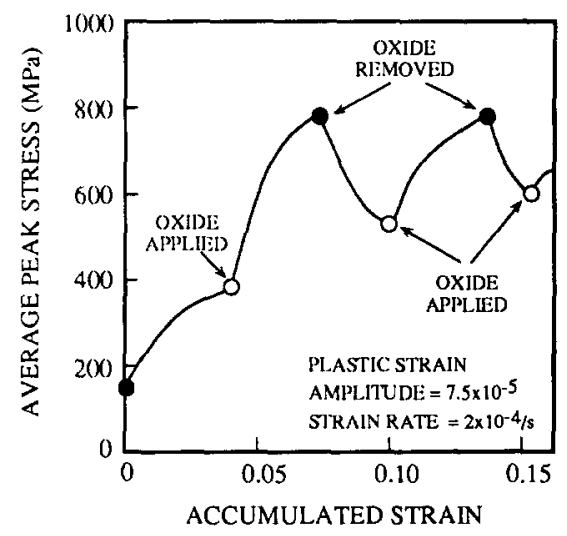

Fig. 3. Effect of successive film applications and removals on cyclic hardening of $\mathrm{W}$ at cyclic microstrains.

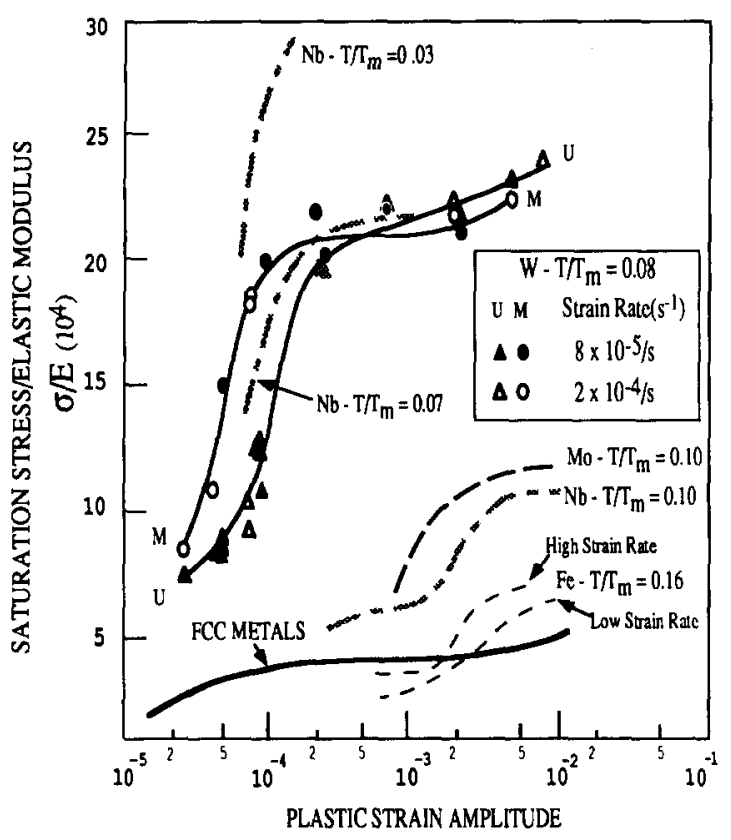

Fig. 2. Modulus-compensated cyclic stress-strain behavior of uncoated (U) and coated (M) ("modified") W compared with that of other bcc metals and fcc metals.

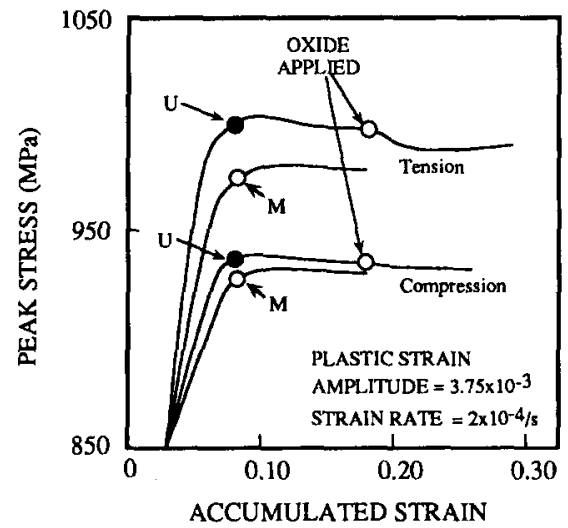

Fig. 4. Effect of film application on cyclic hardening of $\mathrm{W}$ at cyclic microstrains.

\section{$\underline{B}_{2}$ Ordered Alloys.}

Very little research has been done on the fatigue behavior of $\mathrm{B}_{2}$ ordered alloys with low ductility such as $\mathrm{NiAl}$ or FeAl (35), and virtually no work has been done on low-cycle fatigue in plastic strain control $(10,36,37)$. Consequently, little is known conceming the fundamental cyclic deformation behavior and corresponding dislocation substructures of the uncoated materials at low homologous temperatures other than the materials exhibit very little cyclic plasticity, except possibly at very low plastic strain amplitudes. The transition from cyclic microstrain to cyclic macrostrain is also difficult to determine (10). As a result, the baseline cyclic response of these materials cannot be characterized in the broad consistent manner outlined in the previous section for bcc metals. We restrict our discussion of these materials to some very large plasticity enhancement effects of surface films on the rather limited cyclic deformation of polycrystalline $\mathrm{Ni}$ 20Fe-30 Al and $\mathrm{Fe}-40 \mathrm{Al}$ alloys. Both materials exhibit surface-film-enhanced plasticity in monotonic tensile testing for the same Ni films employed in these experiments (36). 
Recently, several types of experiments have demonstrated the sensitivity of monotonic or cyclic piastic flow and toughness of $\mathrm{Ni}-\mathrm{Al}, \mathrm{Ni}-\mathrm{Fe}-\mathrm{Al}$ and $\mathrm{Fe}-\mathrm{Al} \mathrm{B}_{2}$ ordered alloys at room temperature to the initial mobile dislocation density and dislocation generation processes. Enhanced mechanical properties can result from reduced hot extrusion temperatures $(10,36,38)$, room temperature and elevated temperature prestrain $(10,36,39)$, microalloying $(40)$, ductile phase toughening (41), and surface films $(10,11,36)$. Some of our results on enhanced cyclic plasticity of $\mathrm{Ni}-\mathrm{Fe}-\mathrm{Al}$ and $\mathrm{Fe}-\mathrm{Al}$ alloys from prestrain and surface films are given in Fig. 5.

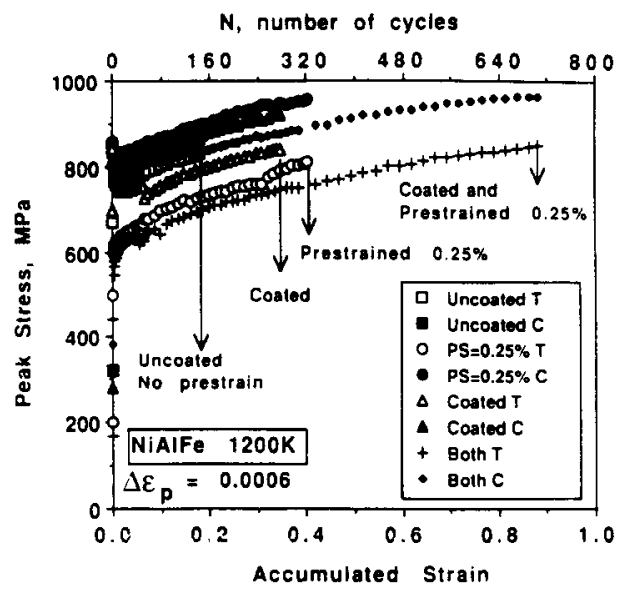

(a)

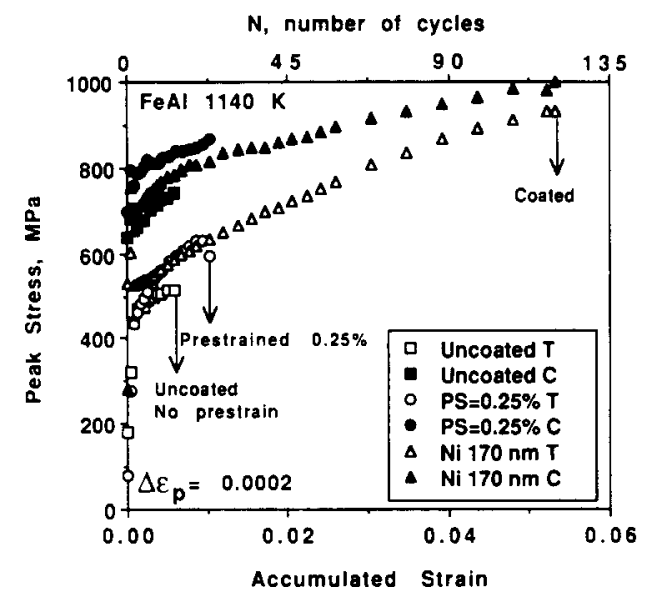

(b)

Fig. 5. Influence of compressive prestrain (PS) and surface films on cyclic deformation of (a) Ni-20Fe-30Al and (b) Fe-40Al at room temperature. Data for both tension (T) and compression (C) cycles are shown. Extrusion temperatures and plastic strain amplitudes $\left(\Delta \varepsilon_{\mathrm{p}}\right)$ are also listed.

Ni-Fe-Al Alloys. The fatigue life of the ternary alloy, which deforms by $<001\rangle$ slip, is increased from approximately $18 \%$ accumulated strain to about $35-40 \%$ by either a small amount $(0.25 \%)$ of compressive prestrain at room temperature or application of a thin $(170 \mathrm{~nm})$ nickel film. Combining prestrain and coating increases the strain to failure to almost $90 \%$. These results are for a material which had been hot extruded at $1200 \mathrm{~K}$. Except for relative magnitudes, similar enhancements are obtained for the same material hot extruded at $1400 \mathrm{~K}$. The latter uncoated material is completely brittle and exhibits no monotonic or cyclic plasticity at room temperature, but can sustain a 4-5\% accumulated plastic strain if prestrained or nickel-coated $(10,36)$. The differences in response of these uncoated materials lie in the initial mobile dislocation densities afforded by the choice of extrusion temperatures. In the material extruded at $1400 \mathrm{~K}$ there is only a very small density of mobile $<001>$ dislocations present in the as-extruded state. Hot extrusion at $1200 \mathrm{~K}$ produces a sufficient density of mobile dislocations to accommodate some monotonic and cyclic plasticity. The effect of compressive prestrain and/or application of surface films is to supply more $<001>$ mobile dislocations, initially enabling dislocation multiplication and generation processes that would not be operative in the uncoated materials.

Fe-Al Alloys. The fatigue lives of Fe-40Al alloys, which deform by $<111>$ slip, are generally quite small, with accumulated plastic strains less than $1 \%$, except for boron-doped materials (10). Figure 5 illustrates that the same compressive prestrain and surface-film coating used for the $\mathrm{Ni}-\mathrm{Fe}-\mathrm{Al}$ alloys can enhance the cyclic plasticity to as much as $5 \%$. The prestrain effect is probably not very pronounced in the $\mathrm{Fe}-\mathrm{Al}$ alloys because the extrusion temperatures chosen afford an ample initial mobile dislocation density to accommodate some monotonic and cyclic deformation. Thus, introducing new dislocations by prestrain has only a small effect on cyclic deformation. By contrast, the surface films in a bec-like material such as Fe-40Al can generate mobile non-screw dislocations which compensate for the reduced relative mobility of screw dislocations at and below room temperature.

\section{Summary and Conclusions}

This paper demonstrates, as others in the viewpoint set have, that there are large surface effects in the fatigue response of the materials investigated. For dislocation-mobility-limited materials such as bcc metals and $\mathrm{B}_{2}$ ordered alloys, surface films can greatly enhance the monotonic and cyclic plasticity at low homologous temperatures by allowing generation of mobile dislocations into the materials from sources that would not otherwise be available or operative. For bec metals, distinct cyclic film-induced hardening and film-induced softening regimes are observed corresponding to relatively low and high plastic strain amplitudes, respectively. For $\mathrm{B}_{2}$ ordered alloys, film-enhanced cyclic plasticity is also observed, even though the cyclic deformation behavior is not as well characterized. 


\section{Acknowledgements}

Various parts of this research have been supported by the U.S. National Science Foundation, Grant Nos. DMR8506705, DMR-8810058 and DMR-9102414.

\section{References}

1. U.F. Kocks, A.S. Argon and M.F. Ashby, Thermodynamics and Kinetics of Slip, Progress in Materials Science, Vol. 19, Bruce Chalmers, J.W. Christian and T.B. Massalski, eds., Pergamon Press, New York, 1975.

2. J.W. Christian, Met. Trans. 14, 1237 (1983).

3. P.M. Hazzledine and Y.Q. Sun, High-Temperature Ordered Intermetallic Alloys IV, L.A. Johnson, D.P. Pope and J.O. Stiegler, eds., MRS Symposium Proceedings 213, 209 (1991).

4. V.K. Sethi and R. Gibala, Acta Met. 25, 321 (1977).

5. V.K. Sethi and R. Gibala, Phil. Mag. A 37, 419 (1978).

6. J.E. Talia, L. Fernandez and R. Gibala, Scripta Met. 12, 737 (1978).

7. T. Tottori, J.E. Talia and R. Gibala, Scripta Met. 14, 1153 (1980).

8. S. Kobayashi and M. Meshii, Acta Met. 25, 1515 (1977).

9. R.D. Noebe, J.T. Kim, J.W. McVay and R. Gibala, Strength of Metals and Alloys (ICSMA 8), P.O. Kettunen, Y.K. Lepistö and M.E. Lehtonen, eds., Pergamon Press, New York, p. 415, 1988.

10. S.E. Hartfield-Wünch, Ph.D. Thesis, University of Michigan, Ann Arbor, MI (1991).

11. R.D. Noebe and R. Gibala, Scripta Met. 20, 1635 (1986).

12. R.D. Noebe and R. Gibala, Structure and Deformation of Boundaries, K.N. Subramanian and M.A. Imam, eds. The Metallurgical Society, Warrendale, PA, p. 89, 1986.

13. M. Yamaguchi, Mechanical Properties of BCC Metals, M. Meshii, ed., TMS, Warrendale, PA, p. 31, 1982.

14. R.D. Noebe, R.R. Bowman, J.T. Kim, M. Larsen and R. Gibala, High Temperature Aluminides and Intermetallics, S.H. Whang, C.T. Liu, D.P. Pope and J.O. Steigler, eds., The Minerals, Metals and Materials Society, Warrendale, PA., p. 271, 1990.

15. K. Benhaddane and P. Beauchamp, Phys. Stat. Sol. 98, 195 (1986).

16. D. Farkas, R. Pasianot, E.J. Savino and D.B. Miracle, High Temperature Ordered Intermetallic Alloys IV, L.A. Johnson, D.P. Pope and J.O. Stiegler, eds, MRS Symposium Proceedings 213, 223 (1991).

17. J.T. Kim, Ph.D. Thesis, University of Michigan, Ann Arbor, MI, 1990.

18. J.T. Kim, R.D. Noebe and R. Gibala, Intermetallic Compounds - Structure and Mechanical Properties - (JIMIS 6), Osamu Izumi, ed., Japan Institute of Metals, Sendai, Japan, p. 591, 1991.

19. G.E. Ruddle and H.G.F. Wilsdorf, Appl. Phys. Lett. 12, 271 (1968).

20. K.J. Bowman, Ph.D. Thesis, University of Michigan, Ann Arbor, MI 1987.

21. K.J. Bowman and R. Gibala, Scripta Met. 20, 1451 (1986).

22. K.J. Bowman and R. Gibala, Acta Met. Mater. 39 (1991), in press.

23. F. Guiu, J.A. Planell and M. Anglada, Dislocations and Properties of Real Materials, M.H. Loretto, ed., Institute of Metals, London, p. 263, 1985.

24. M. Anglada, B. Etemad, J.A. Planell and F. Guiu, Scripta Met. 14, 1319 (1980).

25. M. Anglada and F. Guiu, Phil. Mag. 44, 499 (1981).

26. H. Mughrabi, Dislocations and Properties of Real Materials, M.H. Loretto, ed., Institute of Metals, London, p. 244,1985 .

27. H. Mughrabi, K. Herz and X. Stark, Int. J. of Fracture 17, 193 (1981).

28. H. Mughrabi, K. Herz and X. Stark, Acta Met., 24, 659 (1976).

29. H. Mughrabi, Dislocations and Properties of Real Materials, edited by M. H. Loretto, Publ. 323, Institute of Metals, London, p. 244 (1985).

30. C.J. McMahon, ed. Microplasticity, Wiley, New York, NY, 1968.

31. Z.S. Basinski, A.S. Korbel and S.J. Basinski, Acta Met. 28, 191 (1980).

32. C. Laird and L. Buchinger, Met. Trans. 16A, 2201 (1985).

33. J.E. Talia and R. Gibala, unpublished research

34. K.J. Bowman, EMSA Proceedings, p. 700, 1986.

35. N.S. Stoloff, G.E. Fuchs, A.K. Kuruvilla and S.J. Choe, High Temperature Ordered Intermetallic Alloys, N.S. Stoloff, C.C. Koch, C.T. Liu and O. Izumi, eds., MRS Symposium Proceedings 81,247 (1987).

36. S.E. Hartfield-Wünsch and R. Gibala, High Temperature Ordered Intermetallic Alloys IV, L.A. Johnson, D.P. Pope and J.O. Stiegler eds., MRS Symposium Proceedings 213, 575 (1991).

37. K.B. Bain, R.D. Field and D.F. Lahrman, Symposium on Physical Metallurgy of Intermetallic Compounds, TMS Fall Meeting, 1991.

38. S. Guha, P. Munroe and I. Baker, Scripta Met. 23, 897 (1989).

39. J.E. Hack, T.-Y. Zhang, J.M. Brzeski and R. Darolia, Symposium on Physical Metallurgy of Intermetallic Compounds, TMS Fall Meeting, 1991.

40. R. Darolia, J. Metals, Vol. 43, No. 3, p. 44, 1991.

41. A. Misra, S.E. Hartfield-Wünsch and R. Gibala, Intermetallic Compounds - Structure and Mechanical Properties (JIMIS-6), Osamu Izumi, ed., Japan Institute of Metals, Sendai, Japan, p. 597, 1991. 